\title{
Medium- and high-spin band structure of the chiral candidate ${ }^{132} \mathrm{La}$
}

I. Kuti, ${ }^{1}$ J. Timár, ${ }^{1}$ D. Sohler, ${ }^{1}$ E. S. Paul, ${ }^{2}$ K. Starosta, ${ }^{3}$ A. Astier, ${ }^{4}$ D. Bazzacco,${ }^{5}$ P. Bednarczyk, ${ }^{6}$ A. J. Boston, ${ }^{2}$ N. Buforn, ${ }^{4}$ H. J. Chantler, ${ }^{2}$ C. J. Chiara, ${ }^{7,8}$ R. M. Clark ${ }^{9}$ M. Cromaz, ${ }^{9}$ M. Descovich, ${ }^{2}$ Zs. Dombrádi, ${ }^{1}$ P. Fallon, ${ }^{9}$ D. B. Fossan, ${ }^{10}$ C. Fox ${ }^{2}$ A. Gizon, ${ }^{11}$ J. Gizon, ${ }^{11}$ A. A. Hecht,${ }^{12}$ N. Kintz, ${ }^{13}$ T. Koike,,${ }^{14}$ I. Y. Lee, ${ }^{9}$ S. Lunardi, ${ }^{5}$ A. O. Macchiavelli, ${ }^{9}$ P. J. Nolan, ${ }^{2}$ B. M. Nyakó, ${ }^{1}$ C. M. Petrache, ${ }^{15}$ J. A. Sampson, ${ }^{2}$ H. C. Scraggs, ${ }^{2}$ T. G. Tornyi, ${ }^{1}$ R. Wadsworth, ${ }^{16}$ A. Walker, ${ }^{2}$ and L. Zolnai ${ }^{1}$

${ }^{1}$ Institute of Nuclear Research, Pf. 51, 4001 Debrecen, Hungary

${ }^{2}$ Oliver Lodge Laboratory, Department of Physics, University of Liverpool, Liverpool L69 7ZE, United Kingdom

${ }^{3}$ Department of Chemistry, Simon Fraser University, Burnaby, British Columbia V5A 1S6, Canada

${ }_{4}^{4}$ Institut de Physique Nucléaire de Lyon, IN2P3-CNRS, F69622 Villeurbanne, France

${ }^{5}$ Dipartimento di Fisica and INFN Sezione di Padova, Padova, Italy

${ }^{6}$ Institut Fizyki Jadrowej PAN, Radzikowskiego 152, 31-342 Krakow, Poland

${ }^{7}$ Physics Division, Argonne National Laboratory, Argonne, Illinois 60439, USA

${ }^{8}$ Department of Chemistry and Biochemistry, University of Maryland, College Park, Maryland 20742, USA

${ }^{9}$ Nuclear Science Division, Lawrence Berkeley National Laboratory, Berkeley, California 94720, USA

${ }^{10}$ Department of Physics and Astronomy, State University of New York, Stony Brook, New York 11794-3800, USA

${ }^{11}$ Institut des Sciences Nucléaires, IN2P3-CNRS, F38026 Grenoble, France

${ }^{12}$ Wright Nuclear Structure Laboratory, Yale University, New Haven, Connecticut 06520, USA

${ }^{13}$ Institut de Recherches Subatomiques, Strasbourg, France

${ }^{14}$ Graduate School of Science, Tohoku University, Sendai 980-8578, Japan

${ }^{15}$ Centre de Spectrométrie Nucléaire et de Spectrométrie de Masse, Université Paris-Sud and IN2P3-CNRS, Bâtiment 104-108, F91405 Orsay, France

${ }^{16}$ Department of Physics, University of York, Heslington YO10 5DD, United Kingdom

(Received 27 November 2012; published 15 April 2013)

\begin{abstract}
Medium- and high-spin states of ${ }^{132} \mathrm{La}$ have been studied based on the data obtained from ${ }^{100} \mathrm{Mo}\left({ }^{36} \mathrm{~S}, p 3 n\right)$ and ${ }^{116} \mathrm{Cd}\left({ }^{23} \mathrm{Na}, \alpha 3 n\right)$ fusion-evaporation reactions using the EUROBALL and Gammasphere detector arrays, respectively. Triple- $\gamma$ coincidence relations, angular correlations, and linear polarizations of the observed $\gamma$ transitions have been deduced. The level scheme of ${ }^{132} \mathrm{La}$ has been considerably extended, and unambiguous spin and parity values have been assigned to most of the excited states. The configuration of one of the bands is $\pi g_{7 / 2}\left(h_{11 / 2}\right)^{2} v h_{11 / 2}$ instead of the previously thought $\pi h_{11 / 2} v h_{11 / 2}$; thus its previously suggested magnetic rotational character cannot be upheld. The observed similarities between the level structures of ${ }^{132} \mathrm{La}$ and ${ }^{134} \mathrm{Pr}$ suggest the possible existence of a third $\pi h_{11 / 2} v h_{11 / 2}$ band in ${ }^{132} \mathrm{La}$ that may show chiral features.
\end{abstract}

DOI: 10.1103/PhysRevC.87.044323

PACS number(s): 21.10.Hw, 21.10.Re, 23.20.Lv, 27.60.+j

\section{INTRODUCTION}

Rotational bands in odd-odd $A \approx 135$ nuclei have attracted great interest since it was suggested that the near degeneracy of the two observed $\pi h_{11 / 2} v h_{11 / 2}^{-1}$ bands in ${ }^{134} \operatorname{Pr}$ [1] could be caused by chiral symmetry breaking [2]. According to the chiral scenario, the angular momentum of the triaxially deformed nuclear core aligns along the intermediate axis, while the angular momenta of the particle-type and the hole-type quasiparticles align along the short and the long axes, respectively, in order to minimize the interaction energy. Thus, a triplet of mutually perpendicular vectors is formed, and this triplet can be oriented either in a left-handed or right-handed way. This leads to the doubling of the rotational bands corresponding to such configurations [2-4]. Besides the near degeneracy, the chiral partner bands are also expected to exhibit similar electromagnetic properties, e.g., similar $B(E 2)$ and $B(M 1)$ reduced transition probabilities. Up to now, several chiral-candidate doublet band structures have been observed in the $A \approx 130$ and $A \approx 105$ mass regions (see, e.g., Ref. [5]) as well as in the $A \approx 190$ and $A \approx 80$ mass regions [6,7].

The first two nuclei where chiral-candidate doublet band structure was observed were ${ }^{134} \operatorname{Pr}[1]$ and ${ }^{132} \mathrm{La} \mathrm{[8].} \mathrm{In} \mathrm{both}$ nuclei, however, Doppler-shift attenuation method (DSAM) lifetime experiments demonstrated that the $B(E 2)$ values in the chiral-candidate doublet bands differ considerably from each other [9-11]. Thus, for these nuclei the chiral scenario does not seem to hold. For ${ }^{134} \mathrm{Pr}$, several new theoretical attempts have been made to explain the nature of the observed doublet structure [9,12-14], but these have led to different conclusions. They all have concentrated on the doublet structure; however, very recently a third $\pi h_{11 / 2} v h_{11 / 2}^{-1}$ band was observed in ${ }^{134} \operatorname{Pr}[15]$ which could be a chiral partner of the previously known $\pi h_{11 / 2} v h_{11 / 2}^{-1}$ side band. This observation may initiate a rethinking of the present explanations.

In ${ }^{132} \mathrm{La}$, a new band has also been observed [16] which is linked by several transitions to the yrast $\pi h_{11 / 2} v h_{11 / 2}^{-1}$ band and has similar $B(E 2)$ reduced transition probabilities to that of the yrast band [10]. However, the spins and parities of the levels in the new band could not be deduced unambiguously due to the lack of statistics in the experiment. Thus, the quasiparticle configuration of this band is ambiguous. It has been tentatively assigned to the $\pi h_{11 / 2} v h_{11 / 2}^{-1}$ configuration [16]. Recently, the observed properties of these three assumed $\pi h_{11 / 2} v h_{11 / 2}^{-1}$ bands in ${ }^{132} \mathrm{La}$ have been described well with the shears 
mechanism with principal axis cranking (SPAC) model as magnetic rotational bands with different planar symmetries and with a remarkable contribution of collectivity [17]. If the above assumption for the configuration of the new band is right, then it can correspond to the third $\pi h_{11 / 2} v h_{11 / 2}^{-1}$ band in ${ }^{134} \mathrm{Pr}$, and according to the similarity between the two nuclei, the three $\pi h_{11 / 2} \nu h_{11 / 2}^{-1}$ bands in ${ }^{134} \mathrm{Pr}$ could also be explained as magnetic rotational bands. However, if the new band has a different configuration, then we can expect the existence of another band in ${ }^{132} \mathrm{La}$ with the $\pi h_{11 / 2} v h_{11 / 2}^{-1}$ configuration, which could be observed in a higher-statistics experiment.

In order to determine the quasiparticle configuration of the new rotational band in ${ }^{132} \mathrm{La}$, we have studied the mediumand high-spin structure of this nucleus in two heavy-ion fusion-evaporation reaction experiments. We describe the experimental details in Sec. II, detail the obtained new level scheme in Sec. III, and discuss the band configurations and the similarity between ${ }^{132} \mathrm{La}$ and ${ }^{134} \mathrm{Pr}$ in Sec. IV.

\section{EXPERIMENTAL METHODS AND RESULTS}

Study of the ${ }^{132} \mathrm{La}$ band structure was carried out based on data obtained from two experiments. In the first experiment, the $\left.{ }^{100} \mathrm{Mo}{ }^{36} \mathrm{~S}, p 3 n\right)$ reaction was used at a bombarding energy of $160 \mathrm{MeV}$. The beam was provided by the Vivitron accelerator at Institut de Recherches Subatomiques (IReS), Strasbourg, with an intensity of approximately $30 \mathrm{enA}$. The target was made of a single $500 \mu \mathrm{g} / \mathrm{cm}^{2}$ self-supporting, enriched ${ }^{100}$ Mo foil. The $\gamma$ rays were detected by the EUROBALL IV spectrometer [18], which consisted of 15 cluster [19], 26 clover [20], and 30 tapered Ge detectors. The cluster detectors were placed at backward angles, and the clover detectors were positioned symmetrically in two rings at near $90^{\circ}$ relative to the beam direction. The data collected with the EUROBALL detector array were used to determine $\gamma$-ray energies, intensities, coincidence relations, and linear-polarization values.

In the other experiment, ${ }^{132} \mathrm{La}$ was populated using the ${ }^{116} \mathrm{Cd}\left({ }^{23} \mathrm{Na}, \alpha 3 n\right)$ fusion-evaporation reaction at a beam energy of $115 \mathrm{MeV}$. The experimental setup consisted of the Gammasphere detector array of 99 large-volume, Comptonsuppressed HPGe detectors in 16 rings covering a large fraction of $4 \pi$. The experiment using the Gammasphere detector system was used to Directional Correllation from Oriented Nuclei (DCO) information in order to assign $\gamma$-ray multipolarities.

In the EUROBALL experiment, approximately $1.5 \times 10^{9}$ four- or higher-fold coincidence events were collected and unfolded into triple coincidences. For the analysis of the triple-coincidence relations, the RADWARE software package has been used [21]. Typical $\gamma \gamma \gamma$-coincidence spectra are presented in Fig. 1. Energies and relative intensities of the $\gamma$ transitions of ${ }^{132} \mathrm{La}$ determined from the $\gamma \gamma \gamma$-coincidence cube are given in Table I. The energy and intensity calibration of the Ge detectors were made by using ${ }^{133} \mathrm{Ba}$ and ${ }^{152} \mathrm{Eu}$ radioactive sources. The systematic errors due to the energy and efficiency calibrations were estimated to be $\sim 0.2 \mathrm{keV}$ and $\sim 5 \%$, respectively. A total of 107 transitions were detected in the studied nucleus, almost one quarter of which have been assigned to it in the present work.

In order to obtain information on the $\gamma$-ray multipolarities of ${ }^{132} \mathrm{La}$, angular-correlation information [22] was extracted from the data collected in the Gammasphere experiment. For the DCO analysis, we used the three most forward and

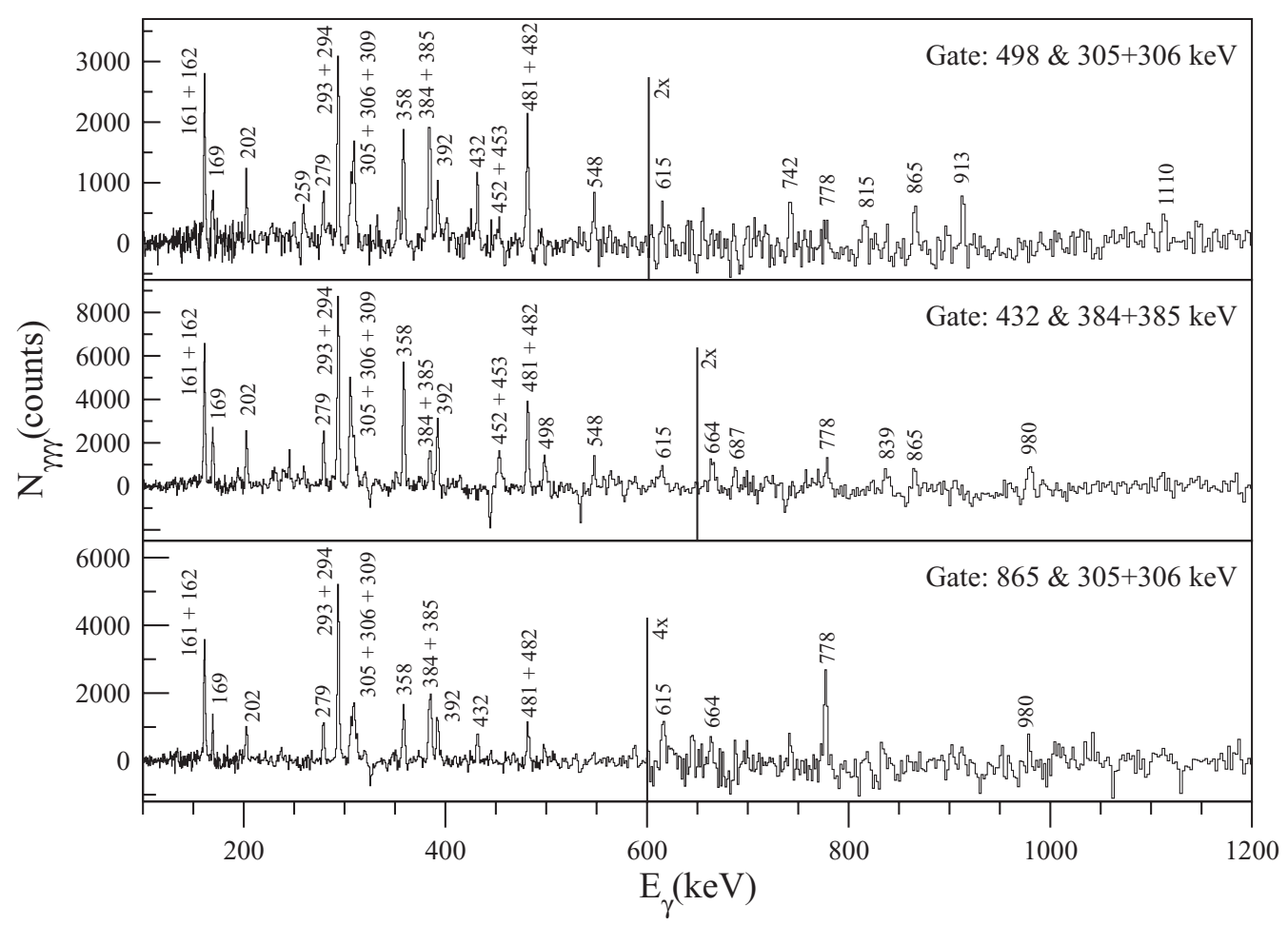

FIG. 1. Typical $\gamma \gamma \gamma$-coincidence spectra obtained in the present work showing the placement of the $\gamma$ rays in band 3 . 
TABLE I. Energies, relative intensities, DCO ratios, polarization values (Pol.), multipolarities (Mult.), and the spin-parities and energies of initial levels of $\gamma$ rays assigned to ${ }^{132} \mathrm{La}$. $R_{\text {DCO }}$ values are obtained from dipole gates unless noted otherwise.

\begin{tabular}{|c|c|c|c|c|c|c|}
\hline$E_{\gamma}$ & $I_{\gamma}$ & $R_{\mathrm{DCO}}$ & Pol. & Mult. & $I_{i}^{\pi}$ & $E_{i}$ \\
\hline $33.5(8)^{\mathrm{a}}$ & $1.2(8)^{\mathrm{a}}$ & & & & $7^{-}$ & 391 \\
\hline $8.1(5)^{\mathrm{a}}$ & $28.3(11)^{\mathrm{a}}$ & & & & $8^{+}$ & 708 \\
\hline $66.8(5)$ & $27.4(11)$ & & & $M 1$ & $9^{+}$ & 775 \\
\hline $96.5(5)$ & $6.6(5)$ & $0.42(8)^{\mathrm{b}}$ & & $M 1$ & $4^{-}$ & 232 \\
\hline $117.2(5)$ & $1.7(5)$ & & & & $6^{+}$ & 508 \\
\hline $129.6(8)$ & $4.5(6)$ & $0.46(12)^{\mathrm{b}}$ & & $M 1$ & $5^{-}$ & 362 \\
\hline $131.3(6)$ & $3.4(6)$ & $0.81(13)^{\mathrm{b}}$ & & $\mathrm{D}+\mathrm{Q}$ & $6^{-}$ & 522 \\
\hline $135.5(5)$ & $8.5(6)$ & $0.85(10)^{\mathrm{b}}$ & & $\mathrm{D}+\mathrm{Q}$ & $3^{-}$ & 136 \\
\hline $150.4(3)$ & $14.2(7)$ & & & & $6^{+}$ & 508 \\
\hline $160.5(5)$ & $9.4(6)$ & $0.67(6)^{b}$ & & $M 1$ & $6^{-}$ & 522 \\
\hline $160.7(3)$ & $97.2(2)$ & $1.11(5)$ & $-0.36(26)^{\mathrm{c}}$ & $M 1$ & $10^{+}$ & 936 \\
\hline $161.5(3)$ & $18.9(9)$ & $1.13(6)$ & $-0.36(26)^{\mathrm{c}}$ & $M 1$ & $7^{+}$ & 670 \\
\hline $164.5(5)$ & $3.3(6)$ & $0.63(10)^{b}$ & & $\mathrm{D}$ & $6^{-}$ & 522 \\
\hline $168.5(8)^{\mathrm{d}}$ & $0.9(6)$ & & & & $7^{-}$ & 753 \\
\hline 169.1(3) & $56.6(7)$ & $0.65(25)^{b}$ & $-0.50(30)$ & $M 1$ & $7^{-}$ & 358 \\
\hline $173.4(5)$ & $4.8(6)$ & $0.48(6)^{\mathrm{b}}$ & & $\mathrm{D}$ & $5^{-}$ & 362 \\
\hline $190.5(8)$ & $0.7(5)$ & $0.93(5)$ & & $\mathrm{D}$ & $9^{+}$ & 776 \\
\hline $202.5(3)$ & $47.2(5)$ & $0.47(8)^{b}$ & $-0.30(20)$ & $M 1$ & $7^{-}$ & 391 \\
\hline $227.3(3)$ & $7.6(5)$ & & & & $8^{(-)}$ & 585 \\
\hline $230.6(4)$ & $19.8(7)$ & $0.41(5)^{\mathrm{b}}$ & $-0.35(21)$ & $M 1$ & $7^{-}$ & 753 \\
\hline $242.0(8)$ & $2.6(6)$ & $1.07(10)$ & $-0.73(46)$ & $M 1$ & $13^{-}$ & 2907 \\
\hline & $4.6(9)$ & & & & $\left(7^{-}\right)$ & 776 \\
\hline $258.7(4)$ & $5.3(8)$ & $0.88(15)$ & $-0.10(37)$ & $M 1$ & $14^{-}$ & 3166 \\
\hline 278.7(3) & $44.3(13)$ & $1.96(11)$ & $-0.21(15)$ & $E 1^{\mathrm{e}}$ & $7^{+}$ & 670 \\
\hline $288.6(5)$ & $4.5(6)$ & & & & $9^{(-)}$ & 874 \\
\hline 288.7(10) & $0.5(6)$ & & & & $11^{(-)}$ & 1572 \\
\hline 293.3(5) & $100.0(21)$ & $0.87(9)^{\mathrm{c}}$ & $-0.17(5)^{\mathrm{c}}$ & $M 1$ & $11^{+}$ & 1229 \\
\hline $293.8(5)$ & $84.9(23)$ & $0.87(9)^{\mathrm{c}}$ & $-0.17(5)^{\mathrm{c}}$ & $M 1$ & $12^{+}$ & 1523 \\
\hline $305.2(5)$ & $10.4(7)$ & $1.05(9)$ & $-0.29(23)^{\mathrm{c}}$ & $M 1$ & $17^{-}$ & 4087 \\
\hline $306.5(5)$ & $8(7)$ & $1.13(11)$ & -0.29 & $M 1$ & $15^{-}$ & 3472 \\
\hline $309.3(5)$ & $9.6(7)$ & $1.13(11)$ & $-0.63(4$ & $M 1$ & $16^{-}$ & 3781 \\
\hline $312.3(3)$ & $16.6(8)$ & $1.98(12)$ & -0.45 & $E 1^{\mathrm{e}}$ & $7^{+}$ & 670 \\
\hline $319.9(3)$ & $39.6(5)$ & $1.99(11)$ & $-0.34(26)$ & $E 1^{\mathrm{e}}$ & $6^{+}$ & 508 \\
\hline $334.2(5)$ & $4.3(6)$ & $1.04(32)^{\mathrm{b}}$ & & $E 1^{\mathrm{e}}$ & & 522 \\
\hline $350.4(4)$ & 11.8(9) & $1.05(7)$ & $0.8(6)$ & $E 1$ & $8^{+}$ & 708 \\
\hline $358.5(4)$ & $14.2(6)$ & $0.91(9)$ & $-0.59(35)$ & $M 1$ & $18^{-}$ & 4445 \\
\hline $360.8(9)$ & 4.3(10) & & & & $12^{+}$ & 1916 \\
\hline $368.7(9)^{\mathrm{d}}$ & $1.9(7)$ & & & & $\left(10^{-}\right)$ & 1536 \\
\hline $380.2(6)$ & $3.6(5)$ & & & & $13^{+}$ & 2296 \\
\hline $380.2(8)$ & $0.9(5)$ & & & & $10^{(-)}$ & 1254 \\
\hline $383.9(3)$ & $12.6(6)$ & & & & $19^{-}$ & 4829 \\
\hline $385.2(3)$ & $54.7(9)$ & $0.84(10)$ & $-0.10(6)$ & $M 1$ & $14^{+}$ & 2301 \\
\hline $391.2(4)$ & $5.3(7)$ & & & & $9^{-}$ & 1167 \\
\hline 392.1(3) & $71.7(7)$ & $0.97(5)$ & $-0.19(7)$ & $M 1$ & $13^{+}$ & 1915 \\
\hline 393.1(8) & $1.6(5)$ & & & & $\left(19^{-}\right)$ & 4948 \\
\hline $403.8(10)$ & $4.3(9)$ & & & & $14^{+}$ & 2700 \\
\hline $409.5(10)$ & $0.3(5)$ & & & & $10^{(-)}$ & 1283 \\
\hline $414.0(3)$ & $29.7(7)$ & $1.14(14)^{\mathrm{b}}$ & & $\mathrm{Q}$ & $9^{-}$ & 1167 \\
\hline $427.0(5)$ & $4.7(7)$ & $1.25(26)$ & & $\mathrm{D}$ & $15^{+}$ & 3127 \\
\hline $432.1(5)$ & $11.9(6)$ & $0.96(11)$ & $-0.35(20)$ & $M 1$ & $20^{-}$ & 5261 \\
\hline $447.9(5)$ & $5.7(7)$ & $0.54(11)^{\mathrm{b}}$ & $-0.31(17)$ & $M 1$ & $12^{-}$ & 2172 \\
\hline $452.2(5)$ & $26.4(6)$ & $0.96(6)^{\mathrm{c}}$ & $-0.30(11)^{\mathrm{c}}$ & $M 1$ & $16^{+}$ & 3206 \\
\hline $453.4(3)$ & $41.5(8)$ & $0.96(6)^{\mathrm{c}}$ & $-0.30(11)^{\mathrm{c}}$ & $M 1$ & $15^{+}$ & 2754 \\
\hline & $3.6(6)$ & & & & $\left(20^{-}\right)$ & 5411 \\
\hline 481.4(5) & $8.0(5)$ & & & & $21^{-}$ & 5742 \\
\hline
\end{tabular}

TABLE I. (Continued.)

\begin{tabular}{|c|c|c|c|c|c|c|}
\hline$E_{\gamma}$ & $I_{\gamma}$ & $R_{\mathrm{DCO}}$ & Pol. & Mult. & $I_{i}^{\pi}$ & $E_{i}$ \\
\hline $481.5(3)$ & $23.0(10)$ & $1.06(9)$ & $0.22(13)$ & $E 1$ & $7^{+}$ & 670 \\
\hline $487.5(5)$ & $11.8(5)$ & $1.09(14)$ & $-0.48(22)$ & $M 1$ & $18^{+}$ & 4200 \\
\hline 498.1(8) & $7.5(5)$ & $0.92(15)$ & $-0.51(20)$ & $M 1$ & $22^{-}$ & 6241 \\
\hline $506.6(4)$ & $18.9(8)$ & $0.99(6)^{b}$ & $-0.29(27)$ & $M 1$ & $17^{+}$ & 3713 \\
\hline $516.9(5)$ & $5.0(5)$ & & & & $\left(18^{-}\right)$ & 4554 \\
\hline $519.9(10)$ & $4.9(6)$ & $0.59(15)^{\mathrm{b}}$ & & $\mathrm{D}$ & $14^{-}$ & 2912 \\
\hline $547.5(5)$ & $5.7(5)$ & $0.91(20)$ & $-0.37(18)$ & $M 1$ & $23^{-}$ & 6788 \\
\hline $556.8(3)$ & $33.0(8)$ & $1.82(22)$ & $0.24(14)$ & $E 2$ & $11^{-}$ & 1724 \\
\hline $559.0(7)$ & $6.1(5)$ & $0.92(16)$ & $-0.30(21)$ & $M 1$ & $19^{+}$ & 4759 \\
\hline $563.4(10)$ & $3.2(5)$ & & & & $24^{-}$ & 7351 \\
\hline $564.8(5)$ & $8.9(7)$ & $0.44(9)^{\mathrm{b}}$ & & $\mathrm{D}$ & $7^{-}$ & 753 \\
\hline $572.6(5)$ & $6.3(5)$ & & & & $\left(16^{-}\right)$ & 3735 \\
\hline $574.6(6)$ & $1.4(6)$ & $2.14(28)$ & $-0.40(28)$ & $E 1^{\mathrm{e}}$ & $16^{-}$ & 3781 \\
\hline $587.4(4)$ & 20.8(9) & $1.91(11)$ & $0.32(32)$ & $E 2$ & $12^{+}$ & 1523 \\
\hline $614.9(6)$ & $2.4(5)$ & $1.79(14)$ & & $\mathrm{Q}$ & $16^{-}$ & 3781 \\
\hline $615.0(6)$ & $4.7(5)$ & $1.76(15)$ & & Q & $17^{-}$ & 4087 \\
\hline $621.6(12)$ & $4.7(9)$ & & & & $11^{+}$ & 1556 \\
\hline $636.5(8)^{d}$ & $1.9(7)$ & & & & $12^{-}$ & 2172 \\
\hline $664.3(10)$ & $1.9(6)$ & & & & $18^{-}$ & 4445 \\
\hline $667.8(4)$ & $27.4(9)$ & $1.04(10)^{b}$ & $0.62(26)$ & $E 2$ & $13^{-}$ & 2392 \\
\hline $668.7(5)$ & $2.7(5)$ & & & & $10^{(-)}$ & 1254 \\
\hline $686.7(4)$ & $17.0(8)$ & $2.01(13)$ & $0.32(20)$ & $E 2$ & $13^{+}$ & 1915 \\
\hline $687.4(15)$ & $1.6(9)$ & & & & $12^{+}$ & 1916 \\
\hline 699.4(12) & $4.3(5)$ & & & & $11^{(-)}$ & 1573 \\
\hline $718.5(6)$ & $4.3(6)$ & $2.22(45)$ & & $E 1^{\mathrm{e}}$ & $15^{-}$ & 3472 \\
\hline $739.9(8)$ & $4.0(6)$ & & & & $14^{-}$ & 2912 \\
\hline $742.3(5)$ & $2.9(7)$ & & & & $19^{-}$ & 4829 \\
\hline $770.5(6)$ & $21.5(7)$ & $0.97(10)^{b}$ & $0.84(18)$ & $E 2$ & $15^{-}$ & 3162 \\
\hline $772.8(12)$ & $3.6(9)$ & $0.91(6)$ & & $\mathrm{D}$ & $13^{+}$ & 2296 \\
\hline $777.6(4)$ & $33.5(6)$ & 1.91(11) & $0.35(28)$ & $E 2$ & $14^{+}$ & 2301 \\
\hline $785.1(5)$ & $6.6(8)$ & $0.79(9)$ & & $\mathrm{D}$ & $14^{+}$ & 2700 \\
\hline $815.4(6)$ & $3.4(10)$ & $2.29(31)$ & & Q & $20^{-}$ & 5261 \\
\hline 822.7(10) & $4.7(7)$ & & & & $\left(16^{-}\right)$ & 3735 \\
\hline $838.7(4)$ & $22.6(8)$ & $2.08(14)$ & $0.44(28)$ & $E 2$ & $15^{+}$ & 2754 \\
\hline $841.4(11)$ & $4.5(6)$ & & & & $\left(13^{-}\right)$ & 2414 \\
\hline $864.9(8)$ & $8.7(6)$ & $2.08(19)$ & $-0.50(20)$ & $E 1^{\mathrm{e}}$ & $14^{-}$ & 3166 \\
\hline $875.2(5)$ & $15.1(7)$ & $1.01(16)^{\mathrm{b}}$ & & $\mathrm{Q}$ & $17^{-}$ & 4037 \\
\hline $906.3(7)$ & $23.2(6)$ & $2.13(19)^{\mathrm{b}}$ & $0.42(27)$ & $E 2$ & $16^{+}$ & 3206 \\
\hline $910.5(6)$ & $6.0(5)$ & & & & $\left(19^{-}\right)$ & 4948 \\
\hline $913.2(8)$ & $5.4(6)$ & $1.81(21)$ & & $\mathrm{Q}$ & $21^{-}$ & 5742 \\
\hline $958.6(3)$ & $12.1(6)$ & $1.68(27)$ & & Q & $17^{+}$ & 3713 \\
\hline $975.0(12)$ & $4.0(5)$ & & & & $\left(15^{-}\right)$ & 3389 \\
\hline $979.6(8)$ & $4.8(5)$ & $1.72(26)$ & $0.39(25)$ & $E 2$ & $22^{-}$ & 6241 \\
\hline $992.0(12)$ & $6.8(7)$ & $2.21(18)$ & & $E 1^{\mathrm{e}}$ & $13^{-}$ & 2907 \\
\hline $994.2(7)$ & $14.2(6)$ & $0.80(26)^{b}$ & $0.67(48)$ & $E 2$ & $18^{+}$ & 4200 \\
\hline $1018.9(10)$ & $9.4(6)$ & $1.9(19)$ & $0.65(64)$ & $E 2$ & $20^{+}$ & 5219 \\
\hline $1045.6(6)$ & $3.6(5)$ & & & & $23^{-}$ & 6788 \\
\hline $1046.4(10)$ & $9.0(7)$ & $2.06(15)$ & $0.44(35)$ & $E 2$ & $19^{+}$ & 4759 \\
\hline $1068.3(20)$ & $0.8(7)$ & & & & $13^{+}$ & 2296 \\
\hline $1110.5(8)$ & $4.5(6)$ & & & & $24^{-}$ & 7351 \\
\hline 1119.2(9) & $9.4(5)$ & $2.28(35)$ & $0.61(32)$ & $E 2$ & $22^{+}$ & 6338 \\
\hline $1142.4(15)$ & $2.1(6)$ & & & & $12^{-}$ & 2665 \\
\hline
\end{tabular}

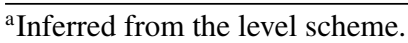

${ }^{\mathrm{b}}$ Obtained from a quadrupole gate.

${ }^{\mathrm{c}}$ Transitions where we could not get separate data for the $\gamma$ rays having nearly the same $E_{\gamma}$ values.

${ }^{\mathrm{d}}$ Tentative assignments.

${ }^{\mathrm{e}} \Delta I=0$ transitions. 
the three most backward detector rings of the Gammasphere detector array against the three rings around $90^{\circ}$. In order to carry out DCO analysis for the $\gamma$ rays, the collected events were sorted into background-subtracted gated spectra using the BLUE software $[23,24]$. In the geometry used, $R_{\mathrm{DCO}}$ values of $\sim 1.0$ and $\sim 0.5$ are expected for stretched quadrupole and stretched dipole transitions, respectively, when a gate is set on a stretched quadrupole transition. Using a stretched dipole gate, the expected values are $\sim 2.0$ and $\sim 1.0$ for stretched quadrupole and stretched dipole transitions, respectively. The expected $R_{\mathrm{DCO}}$ values for the nonstretched $\delta \approx 0$ dipole transitions are about the same as the ones for the stretched quadrupole transitions. We obtained DCO ratios for more than half of the transitions. The DCO ratios determined in the present work are given in Table I.

The multipolarity assignments were further corroborated by extracting the electromagnetic character of the transitions by measuring the linear polarization of the $\gamma$ rays. For this purpose, the four-element clover detectors of the EUROBALL detector array placed close to $90^{\circ}$ relative to the beam direction were used as Compton polarimeters [25]. Two matrices were constructed from $\gamma \gamma$ events; single hits in any detectors were placed on one axis while the added-back double-hit scattering events were placed on the other axis. In the first (second) matrix the scattering events took place perpendicular (parallel) to the reaction plane. The number of perpendicular $\left(N_{\perp}\right)$ and parallel $\left(N_{\|}\right)$scatters for a given $\gamma$ ray were obtained from spectra gated on the single-hit axis of the respective matrix by transitions in coincidence with the given $\gamma$ ray. By assuming that each clover crystal has equal efficiency, an experimental linear polarization is defined as

$$
P=\frac{1}{Q} \frac{N_{\perp}-N_{\|}}{N_{\perp}+N_{\|}},
$$

where $Q$ is the polarization sensitivity for the clover detectors, which is a function of the $\gamma$-ray energy [25]. Strictly speaking, the counting rates should be normalized by a geometrical factor $a\left(E_{\gamma}\right)$, which corrects for the intrinsic asymmetry of the polarimeter. The value of $a\left(E_{\gamma}\right)$ is very close to 1 [1.03(2)] for the EUROBALL clover detectors independently of the $\gamma$-ray energy [20]; thus we accepted 1 for its value. $P>0$ is characteristic for stretched $E 1, E 2$, and nonstretched $M 1$ transitions, while $P<0$ characterizes the stretched $M 1$ and nonstretched $E 1$ transitions. On the basis of the deduced values and our DCO results, 23 transitions were found to have electric character, while $28 \gamma$ rays showed magnetic behavior. The results of the linear-polarization analysis are summarized in Table I.

The polarization sensitivity considerably decreases at lower $\gamma$-ray energies; hence the linear-polarization values could not be determined for the lower-energy transitions. Therefore, determination of the $M 1$ or $E 1$ character of some of the low-energy transitions was carried out by deriving the internalconversion coefficients of the $\gamma$ rays determined from intensity balances. The obtained $\alpha_{\text {tot }}$ conversion coefficients are given in Table II.

For the multipolarity determination, which was based on the obtained DCO ratios, the linear-polarization results, and the conversion coefficients, only electric and magnetic dipole
TABLE II. Conversion coefficients of four low-energy transitions. The theoretical $\alpha_{M 1}$ and $\alpha_{E 1}$ values are taken from Ref. [26]. The $\alpha_{\exp }$ values are derived with the assumption that the 135.5 - and $160.7-\mathrm{keV}$ transitions are $M 1$.

\begin{tabular}{llll}
\hline \hline$E_{\gamma}(\mathrm{keV})$ & $\alpha_{M 1}$ & $\alpha_{E 1}$ & $\alpha_{\exp }$ \\
\hline 66.8 & 3.45 & 0.71 & $2.84(54)$ \\
96.5 & 1.22 & 0.26 & $1.34(24)$ \\
129.6 & 0.53 & 0.12 & $0.45(15)$ \\
160.5 & 0.29 & 0.06 & $0.36(14)$ \\
\hline \hline
\end{tabular}

(D) and electric quadrupole (Q) transitions were considered. The multipolarities deduced in the present work (listed in Table I) agree with the previously assigned multipolarities $[8,10,27,28]$.

\section{LEVEL SCHEME}

Based on the observed $\gamma \gamma \gamma$-coincidence relations as well as energy and intensity balances we have significantly extended the level scheme of ${ }^{132} \mathrm{La}$. The derived level scheme, plotted in Fig. 2, contains six bands with 63 levels and 107 transitions, of which 22 transitions are assigned to ${ }^{132} \mathrm{La}$ in the present work.

We have inserted two additional levels in band 3 above the state decaying by the 306-keV transition (305-keV transition in Ref. [10]) and extended the band to higher spin by four levels. An additional linking transition $(575 \mathrm{keV})$ between band 3 and band 1 has been observed. We have extended band 1 by another level. The favored signature of band 5 and a few side levels connected to it were observed previously [27]. We have extended band 5 above the previously known $17^{-}$state by three levels. We could extend the previously known sequence of side levels and their connecting transitions to the favored signature sequence of band 5, thus assigning it as the unfavored branch of band 5 .

Probably due to the more symmetrical reactions used in the present analysis, band 2 and the bottom part of band 3 were seen to be weaker than in previous experiments. For this reason we were not able to verify the existence of the previously reported transitions with energies of 501, 564, 827, 863,1101 , and $1178 \mathrm{keV}$ [10], 740, 783, 827, 830, 862, and $916 \mathrm{keV}$ [8], or $455 \mathrm{keV}$ [29]. Otherwise, the placement of the earlier known transitions is mostly in agreement with the previous studies except for some minor differences.

The spin and parity assignments proposed in the present paper are based on the known spin-parity of the low-lying levels and on the derived internal-conversion coefficients, linear-polarization values, and DCO ratios of $\gamma$ rays between the excited states. Definite parity was proposed to a state if $E 1, M 1$, or quadrupole character could be determined for one of the transitions connecting it to a state with known parity. For the spin and parity assignments of the excited states, it was assumed that, in the heavy-ion-induced fusion-evaporation reactions, yrast and near-yrast states are preferably populated. Thus, if several spin values are equally possible, then the maximum possible spin value allowed by the angular-distribution ratios of the transitions was assigned to the states. 


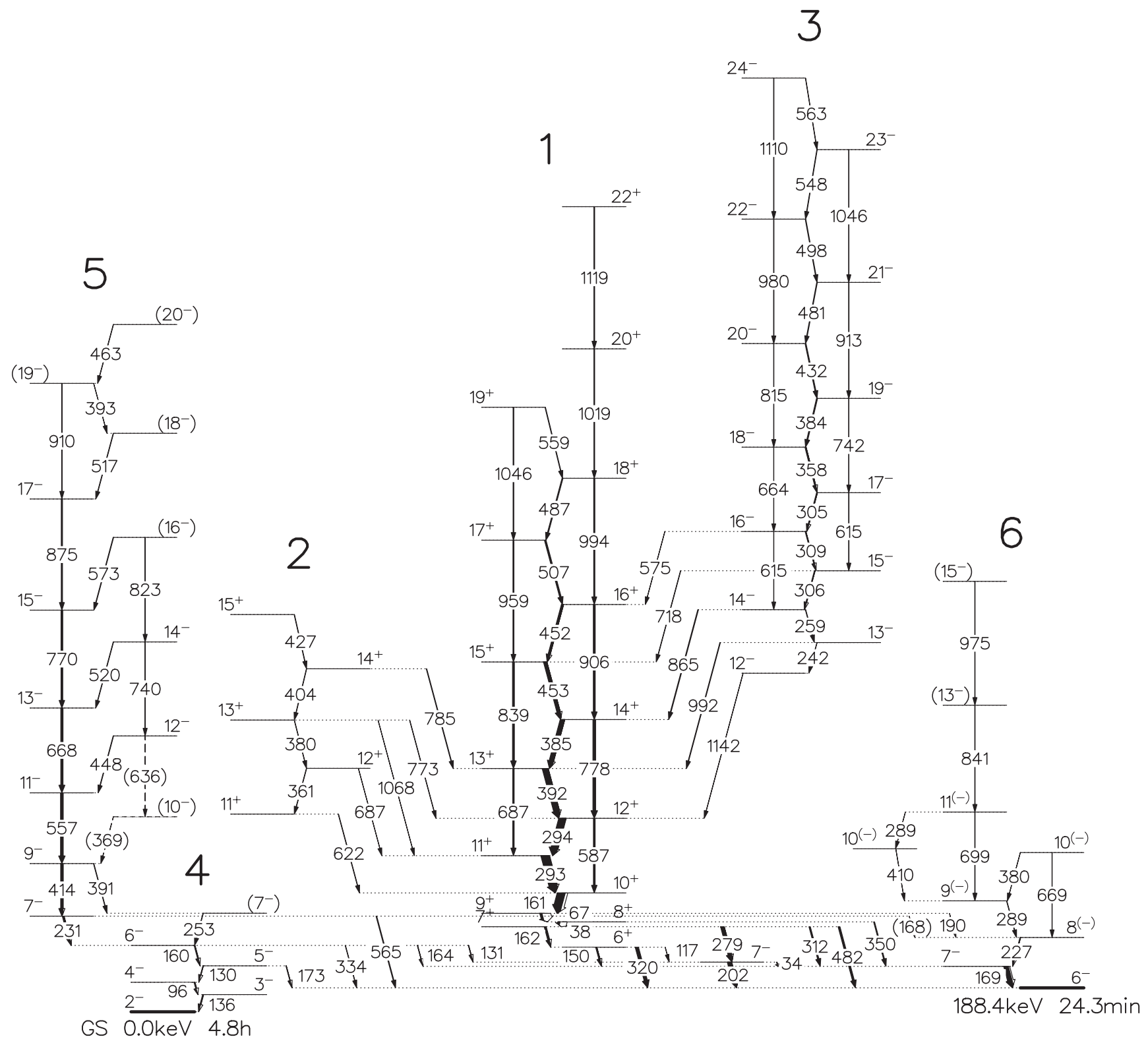

FIG. 2. Medium- and high-spin bands of ${ }^{132} \mathrm{La}$ obtained in the present work. The energies are given in keV, while the widths of the arrows are proportional to the relative transition intensities.

The ground state of ${ }^{132} \mathrm{La}$ has a lifetime of $4.8 \mathrm{~h}$, and it has a spin-parity of $2^{-}$determined from atomic beam magnetic resonance measurements and from $\beta \log f t$ values [30]. A cascade of five transitions was observed decaying to this ground state. Earlier studies assigned dipole multipolarity to these transitions [27] and a stretched $M 1$ character to the $136-\mathrm{keV} \gamma$ ray [31]. Our placement of the known transitions is in agreement with the previous studies. For the 96-, 130-, and $160-\mathrm{keV}$ transitions of band 4 , we could assign $M 1$ character from the internal-conversion coefficients determined by intensity balances. Based on the stretched $M 1$ character of these in-band transitions, we assign $4^{-}, 5^{-}$, and $6^{-}$spinparities to the depopulated levels, respectively.

An isomeric state is known at $188 \mathrm{keV}$, which has a 24.3-min half-life [32]. A spin-parity of $6^{-}$was assigned to it based on the atomic beam magnetic resonance method [32]. We accept the $6^{-}$spin-parity value of the long-lived isomeric state given in Ref. [30]. The second lowest level of band 6 decays to the isomeric state by the $169-\mathrm{keV}$ stretched $M 1$ transition [30]. The obtained 0.65(25) DCO ratio and -0.50(30) linearpolarization value confirm this multipolarity, which allows $5^{-}$ or $7^{-}$spin-parity for the depopulated state. We accepted the $7^{-}$one, because in rotational bands we assign increasing spin values to the levels with increasing excitation energy. We could not get new information on the higher-lying levels of band 6; thus we accepted their spin-parities as they were reported in Ref. [27].

The base state of band 1 decays by a $320-\mathrm{keV} \Delta I=0$ $E 1 \gamma$ transition to the $6^{-}$isomeric state [30]. Our DCO ratio and linear-polarization value show good agreement with this 
assignment. Thus we confirm the $6^{+}$spin-parity of this state. The second level of band 1 decays to the first $7^{-}$state with a $312-\mathrm{keV} \Delta I=0 E 1 \gamma$ ray [30]. Therefore we assign a $7^{+}$spinparity for this state. The $162-\mathrm{keV}$ stretched dipole transition to $6^{+}$and the $482-\mathrm{keV}$ stretched $E 1$ transition to $6^{-}$support this assignment. The $7^{+}$state decays also via a $279-\mathrm{keV}$ transition to a level which has not been associated with any band. We confirmed that this transition has a $\Delta I=0 E 1$ character, and therefore a $7^{-}$spin-parity can be assigned to this level. This shows good agreement with the $M 1$ multipolarity of the 202-keV transition feeding the isomeric state. The third lowest level of band 1 decays by the $350-\mathrm{keV}$ stretched $E 1 \gamma$ ray to the first $7^{-}$state. This allows an $8^{+}$or $6^{+}$spin-parity for the depopulated state. As it is an in-band state decaying to a $7^{+}$band member, we assign $8^{+}$to this state. Both the 67- and 161-keV transitions depopulating the next two levels in this band have stretched $M 1$ multipolarity based on DCO and internal-conversion coefficient results. Therefore $9^{+}$and $10^{+}$spin-parity assignments can be made for these levels. The higher-lying levels of this band are connected via stretched $E 2$ crossover transitions as well as stretched $M 1$ transitions, establishing their spins and parities as they are plotted in Fig. 2. The stretched $M 1$ and stretched $E 2$ characters were confirmed for several transitions by DCO and linear-polarization results, as seen in Table I.

Several levels of the former chiral-candidate band (band 2 in Fig. 2) decay to band 1. Our data confirm the previously known structure of band 2, but we could not add any new information to it. Therefore we accept the band structure as it was reported in Ref. [10].

Band 3 is linked to five states of the yrast band (band 1) at $I^{\pi}=12^{+}-16^{+}$via five transitions. From our DCO analysis and obtained linear-polarization values, both the 575- and $865-\mathrm{keV}$ transitions have $\Delta I=0 E 1$ character. Based on these results we could unambiguously assign $14^{-}$and $16^{-}$ spin-parity to the third and fifth levels of band 3 . The second level of this band is linked to the $14^{-}$state via a $259-\mathrm{keV}$ $M 1 \gamma$ ray, and it decays to the lowest level of the band through a $242-\mathrm{keV}$ transition; therefore we assign $12^{-}$and $13^{-}$ spin-parity to the first and second levels of band 3, respectively. The higher-lying levels of this band are connected via stretched $E 2$ crossover transitions besides the stretched $M 1$ transitions, determining their spins and parities as shown in Fig. 2. This band has been previously reported up to the level which is assigned $20^{-}$in Fig. 2 [10]; in the present work we have extended it up to the $24^{-}$state.

The bandhead of band 5 decays to the $6^{-}$state of band 4 by the $231-\mathrm{keV}$ stretched $M 1$ transition, which allows a $7^{-}$or $5^{-}$spin-parity assignment for this level. Based on the absence of transitions to the $4^{-}$and $3^{-}$states of band 4 , and given the transitions to the lower-lying levels of band 6 , we assign a $7^{-}$spin-parity to the bandhead. This assignment is in good agreement with the previous tentative assignment [27], and it unambiguously establishes the negative parity of this band. The unambiguous spins of the levels of the favored branch up to $17^{-}$are based on the $E 2$ or quadrupole character of the intraband transitions. The $M 1$ character of the $448-\mathrm{keV}$ and stretched dipole character of the 520-keV intraband transitions provide $12^{-}$and $14^{-}$spin-parities for the second and third levels of the unfavored branch of band 5 .

\section{DISCUSSION}

The main aim of the present work was to determine the configuration of the new rotational band of ${ }^{132} \mathrm{La}$ observed by Grodner et al. [16] (band 3 in this work). From the present experiments we could build a new, more complete version of this band and determine unambiguous spins and parities for it. Moreover, we could also determine unambiguous spins and parities for bands 4 and 5. Considering these new experimental observations, we have checked their consistency with the previous configuration assignments and have attempted to assign new configurations to the bands with no previous assignments.

In order to assist in the configuration assignment of the negative-parity band structure, we have derived the experimental alignments $\left(i_{x}\right)$ and Routhians $\left(e^{\prime}\right)$ of the bands as they are defined in Ref. [33] and plotted them in Fig. 3. A rotational reference, with a variable moment of inertia $J_{\text {ref }}=J_{0}+\omega^{2} J_{1}$, has been subtracted with Harris parameters $J_{0}=11 \hbar^{2} \mathrm{MeV}^{-1}$ and $J_{1}=42 \hbar^{4} \mathrm{MeV}^{-3}$. Values of $K=5,6,2,1$, and 6 were assumed for bands $1,3,4,5$, and 6 , respectively. It has been found that the negative-parity band structure of ${ }^{132} \mathrm{La}$ is rather similar to that of its isotone ${ }^{134} \mathrm{Pr}$, recently reported in Ref. [15]. This similarity can be used to assist in the determination of the band configurations in ${ }^{132} \mathrm{La}$, and also to draw further conclusions for the level scheme of this nucleus. Alignments and Routhians of the lowest-lying negative-parity bands, as well as the yrast $\pi h_{11 / 2} v h_{11 / 2}$ and $\pi g_{7 / 2}\left(h_{11 / 2}\right)^{2} v h_{11 / 2}$ bands of ${ }^{134} \mathrm{Pr}$, are also plotted in the figure in order to assist in the comparison of the bands of the two nuclei. For the ${ }^{134} \mathrm{Pr}$ bands, $J_{0}=12 \hbar^{2} \mathrm{MeV}^{-1}$ and $J_{1}=29 \hbar^{4} \mathrm{MeV}^{-3}$ Harris parameters were used.

\section{A. Comparison with the neighboring ${ }^{134} \mathrm{Pr}$}

Comparing the level scheme of ${ }^{132} \mathrm{La}$ with those of the neighboring odd-odd lanthanum nuclei, we can find similarities in the main features, e.g., the existence and doubling of the low-lying $\pi h_{11 / 2} v h_{11 / 2}$ bands and the appearance of a fourquasiparticle band. On the other hand, there are differences in other details, e.g., the ground-state spin-parity and the features of the negative-parity low-lying bands. Comparison with ${ }^{134} \mathrm{Pr}$, however, shows a more pronounced similarity between the negative-parity level schemes of the two nuclei.

In both nuclei, the ground-state spin-parity is $2^{-}$, and a short $\Delta I=1$ band (band 4 in ${ }^{132}$ La and band 12 in ${ }^{134} \operatorname{Pr}[15]$ ) is built on it. Also, in both nuclei a $6^{-}$isomeric state exists close to the ground state with bands built on them. In ${ }^{134} \mathrm{Pr}$, two $\Delta I=1$ bands (bands 9 and 10) are built on this isomeric state and these have similar properties and are linked together by several $\gamma$ transitions. In ${ }^{132} \mathrm{La}$, only one such band (band 6) is observed. However, the poorer statistics of the ${ }^{132} \mathrm{La}$ experiments could lead to only the energetically favored band being observed. The situation is similar with the $\pi h_{11 / 2} v h_{11 / 2}$ bands. In ${ }^{134} \mathrm{Pr}$, three such bands (bands 1, 2, and 3) are observed, while in ${ }^{132} \mathrm{La}$ there are only two (bands 1 and 2 ). Furthermore, in both nuclei there exists a high-spin negative-parity band (band 3 in ${ }^{132} \mathrm{La}$ and band 5 in ${ }^{134} \mathrm{Pr}$ ), which decays to the yrast $\pi h_{11 / 2} v h_{11 / 2}$ band by $\Delta I=0 E 1$ transitions. In ${ }^{134} \mathrm{Pr}$ four high-spin negative-parity bands are reported; the yrast of these behaves similarly to band 3 in ${ }^{132} \mathrm{La}$. 

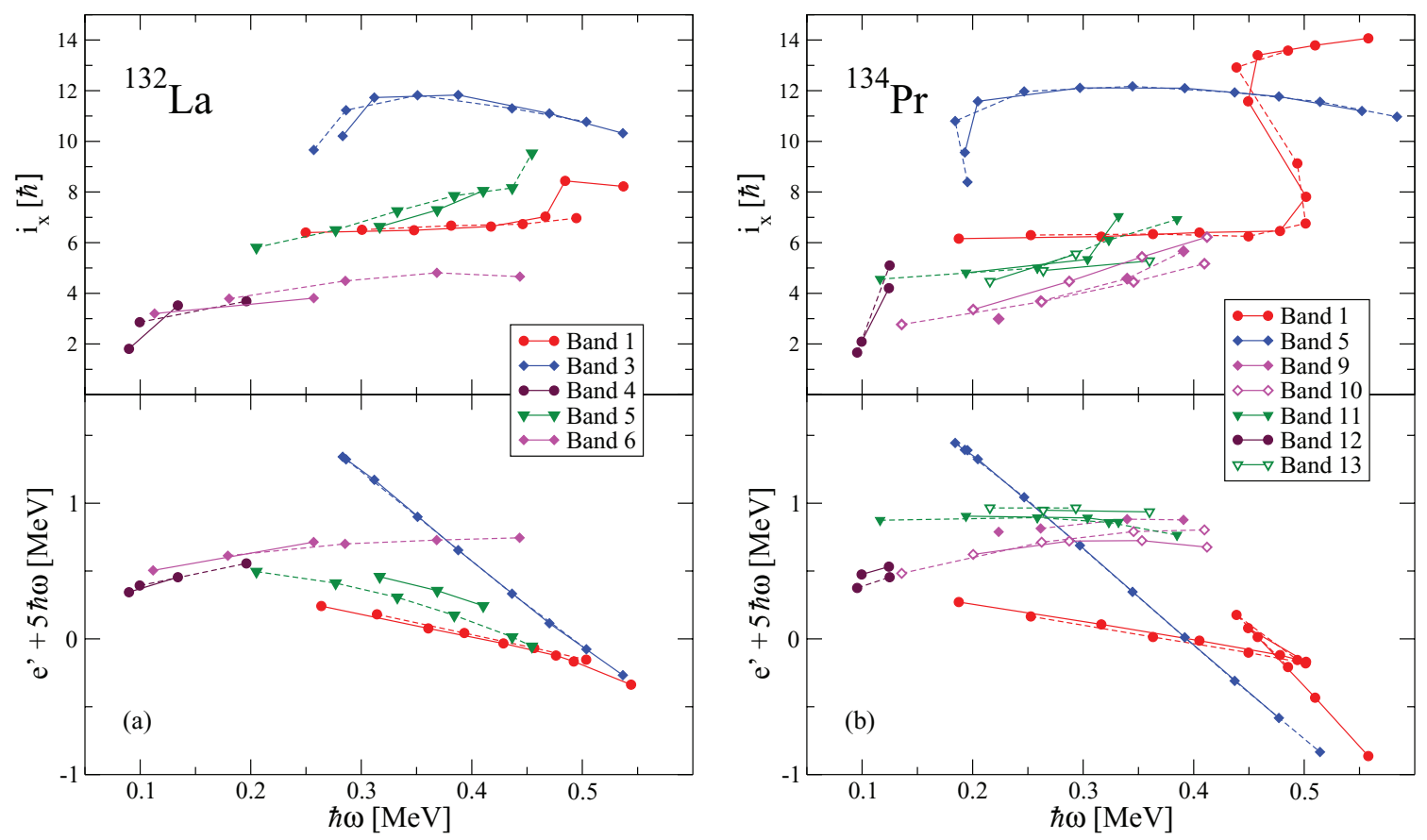

FIG. 3. (Color online) Experimental alignments $\left(i_{x}\right)$ and Routhians $\left(e^{\prime}\right)$ observed in the present work for the ${ }^{132}$ La bands (a) compared with the corresponding values reported for the ${ }^{134} \mathrm{Pr}$ bands (b) in Ref. [15]. Experimental points connected by solid (dashed) lines correspond to the even-spin (odd-spin) branch of the band.

The similarity observed in the global features of these bands in the two nuclei continues to be seen when one inspects their alignments and Routhians as plotted in Fig. 3. Indeed, the alignment and Routhian values of the ${ }^{132} \mathrm{La}$ bands 3, 4, and 6 relative to band 1 are very similar to the values of bands 5, 12, and 10 relative to band 1 in ${ }^{134} \mathrm{Pr}$. However, we can also find differences. In ${ }^{132} \mathrm{La}$, another $\Delta I=1$ negativeparity band (band 5) is observed; it is built on a $7^{-}$state about $600 \mathrm{keV}$ above the $6^{-}$isomeric state. This band shows a large signature splitting with the $\alpha=1$ signature branch being the favored one. In ${ }^{134} \mathrm{Pr}$, there are two bands (bands 11 and 13) which have negative parity and are about $600 \mathrm{keV}$ above the $6^{-}$isomeric state; however, these bands do not show signature splitting. Thus these bands very likely have different configurations. The derived alignment and Routhian values in Fig. 3 are also different for band 5 of ${ }^{132} \mathrm{La}$ and bands 11 and 13 of ${ }^{134} \mathrm{Pr}$.

\section{B. Configurations of the observed bands}

The proton and neutron Fermi levels in ${ }^{132} \mathrm{La}$ are close to the $[550] 1 / 2^{-},[411] 3 / 2^{+}$, and $[413] 5 / 2^{+}$proton states and to the $[514] 9 / 2^{-},[400] 1 / 2^{+}$, and $[404] 7 / 2^{+}$neutron states, respectively. Indeed, the lowest-energy bands in ${ }^{131} \mathrm{La}$ are assigned as based on the rotation-aligned [550]1/2- proton orbital, as well as on the deformation-aligned $[411] 3 / 2^{+}$and [413]5/2 $/ 2^{+}$proton orbitals [34]. In a similar vein in ${ }^{131} \mathrm{Ba}$, the lowest-energy bands are assigned to the $[514] 9 / 2^{-}$and $[400] 1 / 2^{+}$neutron configurations [35]. In the odd-odd ${ }^{132} \mathrm{La}$, we expect bands that are based on the combinations of the above proton and neutron states.

\section{Bands 1 and 2}

The configuration and the possible chiral nature of bands 1 and 2 have been thoroughly discussed in several papers $[4,8,10,17,36]$. According to those results, bands 1 and 2 both have the $\pi h_{11 / 2} v h_{11 / 2}$ configuration; however, they do not form a chiral band structure because of the very different $B(E 2)$ values observed in the two bands. In the present work, no new experimental data which would affect the previous assignments could be derived for these bands. However, the obtained results for band 3 disprove the interpretation suggested in Ref. [17] to understand the nature of bands 1, 2 , and 3. On the other hand, the observed similarity between the level schemes of ${ }^{132} \mathrm{La}$ and ${ }^{134} \mathrm{Pr}$ raises the possibility of the existence of an, as yet unidentified, third band with $\pi h_{11 / 2} v h_{11 / 2}$ configuration close to the two known ones (bands 1 and 2). Such a possible band triplet might be interpreted with the SPAC model as magnetic rotational bands with different planar symmetries. Also, it cannot be ruled out that the possible new $\pi h_{11 / 2} v h_{11 / 2}$ band may form a chiral band structure with one of the already observed ones. Thus, it would be interesting to search in a more sensitive experiment for the existence of such a band having smaller relative intensity.

\section{Band 3}

We have derived negative parity for band 3 . This contradicts the former assignment and hence excludes the $\pi h_{11 / 2} v h_{11 / 2}$ configuration. Furthermore, the observed alignment of this band is $\sim 6 \hbar$ larger than that of band 1 , which shows that it has a four-quasiparticle configuration. Similarity between ${ }^{132} \mathrm{La}$ and ${ }^{134} \mathrm{Pr}$ suggests the $\pi g_{7 / 2}\left(h_{11 / 2}\right)^{2} v h_{11 / 2}$ configuration; 


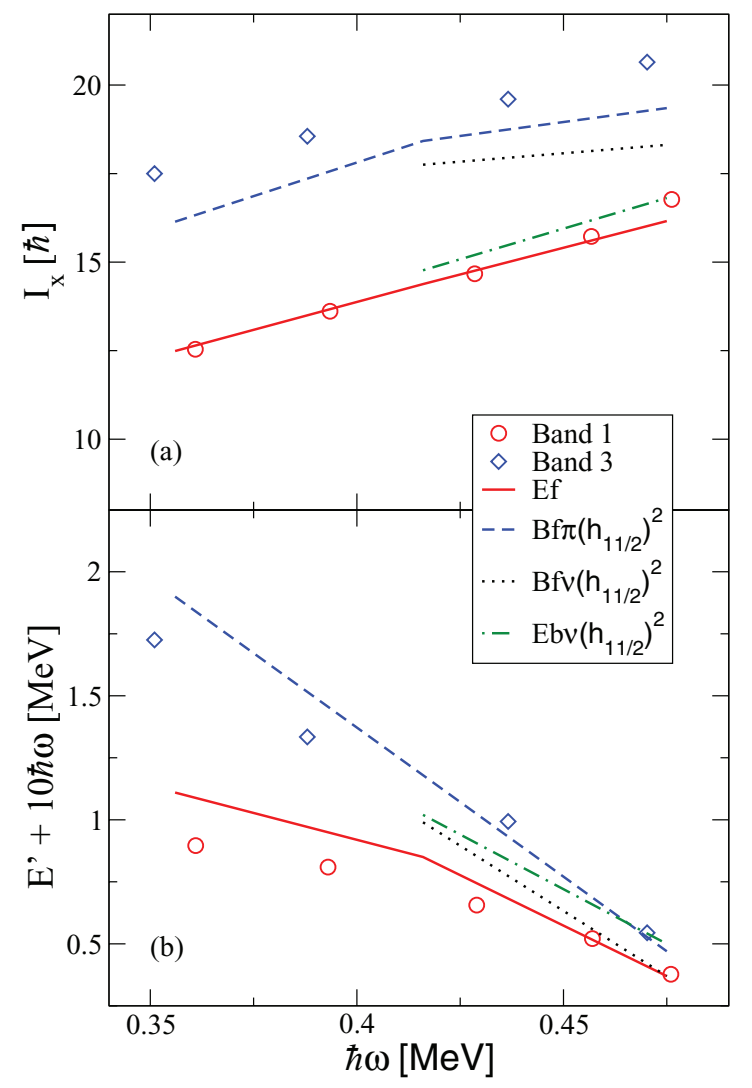

FIG. 4. (Color online) (a) Aligned angular momenta $\left(I_{x}\right)$ and (b) experimental total Routhians $\left(E^{\prime}\right)$ of bands 1 and 3 compared with that of the calculated values for the candidate configurations.

however, the possibility of other negative-parity fourquasiparticle configurations also should be taken into account. Indeed, a similar negative-parity four-quasiparticle band has been found in the neighboring ${ }^{134} \mathrm{La}$ and the $\pi h_{11 / 2} v g_{7 / 2}\left(h_{11 / 2}\right)^{2}$ [37] or $\pi g_{7 / 2} v h_{11 / 2}^{3}$ [38] configuration has been assigned to it. ${ }^{132} \mathrm{La}$ is a $\gamma$-soft nucleus with opposite shape-driving properties of the active particle-type proton and hole-type neutron orbitals; thus the exact nuclear shape strongly influences which of the possible configurations is actually favored.

To assist in the configuration determination, total Routhian surface (TRS) calculations based on the Woods-Saxon cranking formalism have been performed [39-41]. The results of the calculations are compared with the experimental aligned angular momenta and Routhians derived for bands 1 and 3 in Fig. 4. The labeling of the single-quasiparticle orbitals is given in Table III.

The lowest-energy negative-parity four-quasiparticle configurations can be based on the $\mathrm{E}(\mathrm{a}, \mathrm{b})$ or the $\mathrm{B}(\mathrm{e}, \mathrm{f})$ TRS configurations coupled to an aligned pair of $h_{11 / 2}$ protons or $h_{11 / 2}$ neutrons, where $(\mathrm{a}, \mathrm{b})$ and $(\mathrm{e}, \mathrm{f})$ correspond to the two signature branches. Three of these configurations, the $\mathrm{B}(\mathrm{e}, \mathrm{f}) \pi\left(h_{11 / 2}\right)^{2}$, the $\mathrm{B}(\mathrm{e}, \mathrm{f}) v\left(h_{11 / 2}\right)^{2}$, and the $\mathrm{E}(\mathrm{a}, \mathrm{b}) v\left(h_{11 / 2}\right)^{2}$, are predicted by the TRS calculations to appear at lowest energy. Total Routhians $\left(E^{\prime}\right)$ and aligned angular momenta $\left(I_{x}\right)$ of these configurations are compared with the experimental values derived for band 3 in Fig. 4. For the sake of clarity,
TABLE III. Labels used for the quasiproton $(p)$ and quasineutron (n) states of parity $\pi$ and signature $\alpha$.

\begin{tabular}{|c|c|c|c|c|c|}
\hline \multicolumn{3}{|c|}{$p$} & \multicolumn{3}{|c|}{$n$} \\
\hline$\pi, \alpha$ & Label & Shell model & $\pi, \alpha$ & Label & Shell model \\
\hline$(+,+1 / 2)$ & A & $g_{7 / 2}, d_{5 / 2}$ & $(+,+1 / 2)$ & $\mathrm{a}$ & $s_{1 / 2}$ \\
\hline$(+,-1 / 2)$ & B & $g_{7 / 2}, d_{5 / 2}$ & $(+,-1 / 2)$ & $\mathrm{b}$ & $s_{1 / 2}$ \\
\hline$(-,-1 / 2)$ & E & $h_{11 / 2}$ & $(-,-1 / 2)$ & e & $h_{11 / 2}$ \\
\hline$(-,+1 / 2)$ & $\mathrm{F}$ & $h_{11 / 2}$ & $(-,+1 / 2)$ & $\mathrm{f}$ & $h_{11 / 2}$ \\
\hline
\end{tabular}

only one calculated signature branch is plotted for each configuration. The calculated and observed values are also plotted for band 1 , and the experimental Routhians were normalized to the calculated Routhian of band 1 at $\hbar \omega \approx 0.47 \mathrm{MeV}$ by adding the same constant value to the Routhians of the two bands. It is seen that, among the three possible configurations, the $\mathrm{B}(\mathrm{e}, \mathrm{f}) \pi\left(h_{11 / 2}\right)^{2}$ best fits the experimental values of band 3 . Moreover, the calculated signature splitting for this configuration is very small, in agreement with the experimental value, while the calculated signature splittings of the two other configurations are about $400 \mathrm{keV}$. Thus, the comparison with the TRS calculations confirms the configuration assignment suggested by the similarity between ${ }^{134} \mathrm{Pr}$ and ${ }^{132} \mathrm{La}$.

To further strengthen the assignment of the quasiparticle configuration of band 3 , experimental $B(M 1 ; I \rightarrow I-1)$ / $B(E 2 ; I \rightarrow I-2)$ ratios of reduced transition probabilities have been extracted from the measured $I_{\gamma}(M 1) / I_{\gamma}(E 2)$ branching ratios and compared with predictions obtained using the geometrical model of Dönau and Frauendorf [42]. In the calculations, $K_{n}$ and $i_{n}$ were approximated with constant values as listed in Table IV; the appropriate $g_{n}$ values were taken from Ref. [43]. The rotational gyromagnetic factor of $g_{R}=Z / A$ was used in the calculations, while the $Q_{0}$ electric quadrupole moments and the $\gamma$-shape parameters were derived from the nuclear shape predicted by the TRS calculations. The comparison shown in Fig. 5 also confirms the $\pi g_{7 / 2}\left(h_{11 / 2}\right)^{2} v h_{11 / 2}$ configuration for band 3 . The calculated $B(M 1 ; I \rightarrow I-1) / B(E 2 ; I \rightarrow I-2)$ ratios for the other possible configurations are about two orders of magnitude lower than the experimental ones, and therefore they are not plotted in the figure.

\section{Band 4}

Band 4 has very similar characteristics to those of band 12 in ${ }^{134}$ Pr. Indeed, both have similar Routhian and alignment values

TABLE IV. Parameters used for the calculation of $B(M 1) / B(E 2)$ ratios.

\begin{tabular}{lccc}
\hline \hline Configuration & $g$ factor & $K$ & $i_{x}$ \\
\hline$\pi h_{11 / 2}$ & 1.33 & 1.5 & 4.5 \\
$\pi g_{7 / 2}$ & 0.75 & 2.5 & 1.5 \\
$\pi d_{5 / 2}$ & 1.40 & 1.5 & 1.5 \\
$\nu h_{11 / 2}$ & -0.18 & 4.5 & 2 \\
$\nu s_{1 / 2}$ & -1.27 & 0.5 & 0 \\
$\nu g_{7 / 2}$ & 0.3 & 3.5 & 1 \\
\hline \hline
\end{tabular}




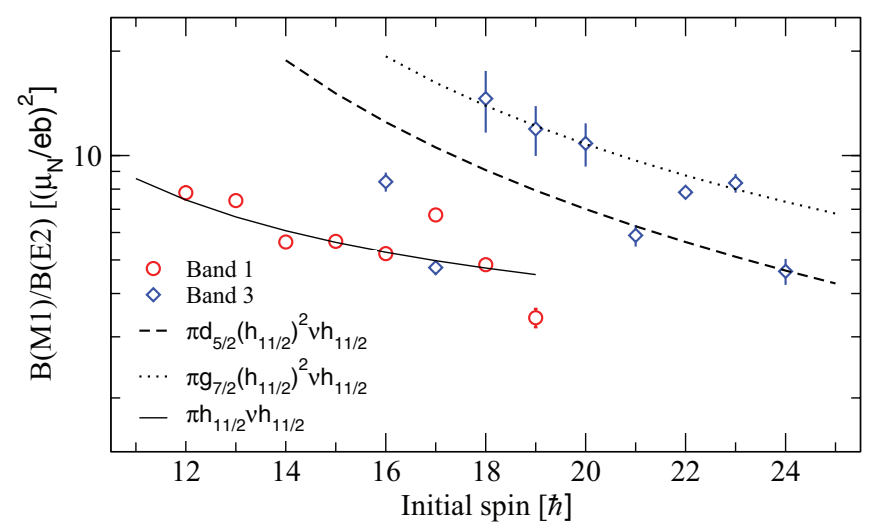

FIG. 5. (Color online) Experimental $B(M 1) / B(E 2)$ ratios of bands 1 and 3 compared with that of the calculated values for the candidate configurations.

and have a $2^{-}$bandhead that is the ground state. Based on this similarity, we expect a $\pi[413] 5 / 2 \nu[514] 9 / 2$ configuration for this band.

The small alignment value for band 4 indicates a twoquasiparticle character. Its negative parity requires involvement of either the proton or the neutron $h_{11 / 2}$ single-particle orbital in the configuration. However, its small alignment value excludes the rotation-aligned proton $h_{11 / 2}$ orbit. Thus, its possible configuration is either $\pi[413] 5 / 2 \nu[514] 9 / 2$ or $\pi[411] 3 /$ $2 v[514] 9 / 2$, both of which contain deformation-aligned orbitals. According to the Gallagher-Moskowski rule [44], the bandhead spin of the first configuration would be $2 \hbar$, while the spin of the second configuration is expected to be $6 \hbar$. Thus, it is a reasonable assumption that band 4 corresponds to the $\pi[413] 5 / 2 v[514] 9 / 2$ configuration similarly to band 12 in ${ }^{134}$ Pr.

\section{Bands 5 and 6}

Unambiguous spin and parity values derived for bands 5 and 6 in the present work confirmed their previous tentative spinparity assignments, according to which both of these bands have negative parity. Their small alignment values indicate two-quasiparticle configurations. Band 6 shows similarity with band 10 in ${ }^{134} \mathrm{Pr}$; however, band 5 has no partner band among the negative-parity bands of ${ }^{134} \mathrm{Pr}$. Indeed, a large $(\approx 200 \mathrm{keV})$ signature splitting is observed for this band, a feature that has not been observed for any of the two-quasiparticle negativeparity bands in ${ }^{134} \mathrm{Pr}$. Furthermore, the alignment of band 5 is considerably larger than that of the possible similar bands in ${ }^{134} \mathrm{Pr}$.

The favored branch of band 5 was first reported in Ref. [27] and a doubly decoupled nature was suggested for it based on the facts that only the odd-spin states of the band were observed and that the level spacings were found to be similar to that of the ground-state band of ${ }^{130} \mathrm{Ba}$. Later, the same group measured the lifetimes of the two lowest transitions [45], from which they determined the $\beta$ deformation parameter. They also could reproduce the doubly-decoupled nature and the experimental level-energy spacings by a pseudospin coupling model involving the proton $\left(h_{11 / 2}, \Omega=1 / 2\right)$ state and the neutron $d_{3 / 2} s_{1 / 2}$ pseudospin doublet. Therefore, we accept this configuration for band 5 .

Band 6 is built on the $6^{-}$isomeric state similarly to band 10 in ${ }^{134} \mathrm{Pr}$. The two bands also have similar Routhian and alignment values. This similarity suggests the $\pi[411] 3 / 2 \nu[514] 9 / 2$ configuration for band 6 . This assignment is also in good agreement with the $\pi g_{7 / 2} \nu h_{11 / 2}$ configuration suggested in Ref. [27].

\section{SUMMARY}

Study of the medium- and high-spin states of ${ }^{132} \mathrm{La}$ has been carried out based on the data obtained in two fusion-evaporation experiments with the EUROBALL and Gammasphere detector arrays. The aim of the present study was to determine the configuration of band 3, part of which was observed previously and tentatively assigned to a $\pi h_{11 / 2} \nu h_{11 / 2}$ configuration magnetic rotational band. We have analyzed the triple- $\gamma$ coincidence relations, DCO angular correlations, and linear polarizations of the observed $\gamma$ transitions. Furthermore, the internal-conversion coefficients of several low-energy transitions also have been derived from intensity balances. As a result of the present work, we have rebuilt the level scheme of ${ }^{132} \mathrm{La}$, inserting new levels into band 3 and extending it to spin 24, as well as extending the other bands. Based on the deduced angular correlations, linear polarizations, and internal-conversion coefficients, we could assign unambiguous spin and parity values to most of the excited states. The new firm energy, spin, and parity values of the levels enabled a more reliable configuration identification, which has been performed by comparing the experimental data with results of calculations and with that of neighboring nuclei. It has been shown that the configuration of band 3 is $\pi g_{7 / 2}\left(h_{11 / 2}\right)^{2} v h_{11 / 2}$ instead of the previously thought $\pi h_{11 / 2} v h_{11 / 2}$; thus the previously suggested $\pi h_{11 / 2} \nu h_{11 / 2}$ magnetic rotational triplet assignment of bands 1, 2, and 3 cannot be held. On the other hand, it has been found that the medium-spin band structure of ${ }^{132} \mathrm{La}$ is quite similar to that of ${ }^{134} \mathrm{Pr}$. Thus, taking into account that three $\pi h_{11 / 2} \nu h_{11 / 2}$ bands have been observed in ${ }^{134} \mathrm{Pr}$, it cannot be ruled out that ${ }^{132} \mathrm{La}$ also has three such bands forming the expected magnetic rotational triplet. Nor can it be ruled out that a possible new $\pi h_{11 / 2} v h_{11 / 2}$ band forms a chiral band structure with one of the already observed ones. Thus, we cannot count the question of chirality in ${ }^{132} \mathrm{La}$ as an answered one. Therefore, it would be interesting to search for the third $\pi h_{11 / 2} v h_{11 / 2}$ band of ${ }^{132} \mathrm{La}$ in a new high-statistics experiment.

\section{ACKNOWLEDGMENTS}

This work was supported in part by the Hungarian Scientific Research Fund, OTKA (Contract No. K100835), the UK Engineering and Physical Sciences Research Council, the Natural Sciences and Engineering Research Council of Canada under Contract No. SAPIN/371656-2010, the Bolyai János Foundation, the US National Science Foundation, as well as the US Department of Energy, Office of Nuclear Physics, under Grant No. DE-FG02-94ER40834. The publication is supported by the TÁMOP-4.2.2/B-10/1-2010-0024 project. The project is co-financed by the European Union and the European Social Fund. 
[1] C. M. Petrache, D. Bazzacco, S. Lunardi, C. Rossi Alvarez, G. de Angelis, M. De Poli, D. Bucurescu, C. A. Ur, P. B. Semmes, and R. Wyss, Nucl. Phys. A 597, 106 (1996).

[2] S. Frauendorf and J. Meng, Nucl. Phys. A 617, 131 (1997).

[3] V. I. Dimitrov, S. Frauendorf, and F. Dönau, Phys. Rev. Lett. 84, 5732 (2000).

[4] K. Starosta et al., Phys. Rev. Lett. 86, 971 (2001).

[5] K. Starosta, AIP Conf. Proc. 764, 77 (2005).

[6] D. L. Balabanski et al., Phys. Rev. C 70, 044305 (2004).

[7] S. Y. Wang et al., Phys. Lett. B 703, 40 (2011).

[8] K. Starosta, C. J. Chiara, D. B. Fossan, T. Koike, T. T. S. Kuo, D. R. LaFosse, S. G. Rohoziński, Ch. Droste, T. Morek, and J. Srebrny, Phys. Rev. C 65, 044328 (2002).

[9] D. Tonev et al., Phys. Rev. Lett. 96, 052501 (2006).

[10] J. Srebrny, E. Grodner, T. Morek, I. Zalewska, Ch. Droste, J. Mierzejewski, A. A. Pasternak, J. Kownacki, and J. Perkowski, Acta Phys. Pol. B 36, 1063 (2005).

[11] E. Grodner, J. Phys.: Conf. Ser. 366, 012022 (2012).

[12] D. Tonev et al., Phys. Rev. C 76, 044313 (2007).

[13] C. M. Petrache, G. B. Hagemann, I. Hamamoto, and K. Starosta, Phys. Rev. Lett. 96, 112502 (2006).

[14] D. Almehed, F. Dönau, and S. Frauendorf, Phys. Rev. C 83, 054308 (2011).

[15] J. Timár et al., Phys. Rev. C 84, 044302 (2011).

[16] E. Grodner, J. Srebrny, Ch. Droste, T. Morek, A. Pasternak, and J. Kownacki, Int. J. Mod. Phys. E 13, 243 (2004).

[17] A. A. Pasternak, E. O. Lieder, and R. M. Lieder, Acta Phys. Pol. B 40, 647 (2009).

[18] J. Simpson, Z. Phys. A 358, 139 (1997).

[19] J. Eberth, H. G. Thomas, P. V. Brentano, R. M. Lieder, H. M. Jäger, H. Kämmerfing, M. Berst, D. Gutknecht, and R. Henck, Nucl. Instrum. Methods Phys. Res. Sect. A 369, 135 (1996).

[20] G. Duchêne, F. A. Beck, P. J. Twin, G. de France, D. Curien, L. Han, C. W. Beausang, M. A. Bentley, P. J. Nolan, and J. Simpson, Nucl. Instrum. Methods A 432, 90 (1999).

[21] D. C. Radford, Nucl. Instrum. Methods A 361, 297 (1995); 361, 306 (1995); http://radware.phy.ornl.gov.

[22] A. Krämer-Flecken, T. Morek, R. M. Lieder, W. Gast, G. Hebbinghaus, H. M. Jäger, and W. Urban, Nucl. Instrum. Methods Phys. Res. Sect. A 275, 333 (1989); K. S. Krane, R. M. Steffen, and R. M. Wheeler, Nucl. Data Tables A 11, 351 (1973).

[23] K. Starosta, D. B. Fossan, T. Koike, C. Vaman, D. C. Radford, and C. J. Chiara, Nucl. Instrum. Methods Phys. Res. Sect. A 515, 771 (2003).

[24] M. Cromaz, T. J. M. Symons, G. J. Lane, I. Y. Lee, and R. W. MacLeod, Nucl. Instrum. Methods A 462, 519 (2001).
[25] P. M. Jones, L. Wei, F. A. Beck, P. A. Butler, T. Byrski, G. Duchêne, G. de France, F. Hannachi, G. D. Jones, and B. Kharraja, Nucl. Instrum. Methods A 362, 556 (1995).

[26] T. Kibédi, T. W. Burrows, M. B. Trzhaskovskaya, P. M. Davidson, and C. W. Nestor Jr., Nucl. Instrum. Methods A 589, 202 (2008).

[27] V. Kumar, P. Das, R. P. Singh, S. Muralithar, and R. K. Bhowmik, Eur. Phys. J. A 17, 153 (2003).

[28] J. Timár et al., Eur. Phys. J. A 16, 1 (2003).

[29] J. R. B. Oliveira, L. G. R. Emediato, M. A. Rizzutto, R. V. Ribas, W. A. Seale, M. N. Rao, N. H. Medina, S. Botelho, and E. W. Cybulska, Phys. Rev. C 39, 2250 (1989).

[30] H. R. Hiddleston and C. P. Browne, Nucl. Data Sheets 17, 225 (1976).

[31] B. Harmatz and T. H. Handley, Nucl. Phys. A 191, 497 (1972).

[32] C. Gerschel and N. Perrin, Compt. Rend. Ser. B 269, 220 (1969).

[33] R. Bengtsson and S. Frauendorf, Nucl. Phys. A 327, 139 (1979).

[34] L. Hildingsson, C. W. Beausang, D. B. Fossan, R. Ma, E. S. Paul, W. F. Piel Jr., and N. Xu, Phys. Rev. C 39, 471 (1989).

[35] R. Ma, Y. Liang, E. S. Paul, N. Xu, D. B. Fossan, L. Hildingsson, and R. A. Wyss, Phys. Rev. C 41, 717 (1990).

[36] E. Grodner et al., Phys. Rev. Lett. 97, 172501 (2006).

[37] R. A. Bark, A. M. Baxter, A. P. Byrne, G. D. Dracoulis, T. Kibédi, T. R. McGoram, and S. M. Mullins, Nucl. Phys. A 691, 577 (2001).

[38] V. Kumar, P. Das, R. P. Singh, R. Kumar, S. Muralithar, and R. K. Bhowmik, Phys. Rev. C 76, 014309 (2007).

[39] W. Nazarewicz, G. A. Leander, and J. Dudek, Nucl. Phys. A 467, 437 (1987).

[40] W. Nazarewicz, R. Wyss, and A. Johnson, Nucl. Phys. A 503, 285 (1989).

[41] R. Wyss, J. Nyberg, A. Johnson, R. Bengtsson, and W. Nazarewicz, Phys. Lett. B 215, 211 (1988).

[42] F. Dönau and S. Frauendorf, in Proceedings of the Conference on High Angular Momentum Properties of Nuclei, edited by N. R. Johnson (Harwood, New York, 1983), p. 143; F. Dönau, Nucl. Phys. A 471, 469 (1987).

[43] A. M. Bizetti-Sona et al., Z. Phys. A 335, 365 (1990); P. Raghavan, At. Data Nucl. Data Tables 42, 189 (1989).

[44] C. J. Gallagher Jr. and S. A. Moszkowski, Phys. Rev. 111, 1282 (1958).

[45] V. Kumar, P. Das, S. Lakshmi, P. K. Joshi, H. C. Jain, R. P. Singh, R. Kumar, S. Muralithar, and R. K. Bhowmik, Phys. Rev. C 82, 054302 (2010). 